\title{
Research on Construction of New-type Low Voltage Distribution Area based on Smart Distribution Transformer Terminal
}

\author{
Shuisheng Lai ${ }^{1, a}$, Lefeng Cheng ${ }^{2, b^{*}}, \underset{e}{\text { Xueli Yang }}{ }^{1, c}$, Yuanmin Lin ${ }^{1, d}$, Zhengjia Li $^{2}$, \\ ${ }^{1}$ Panyu Power Supply Bureau of Guangzhou Power Supply Bureau Co., Ltd, Guangzhou 510000, \\ China \\ ${ }^{2}$ Huatian Power Technology Co., Ltd, Suzhou 215000, China

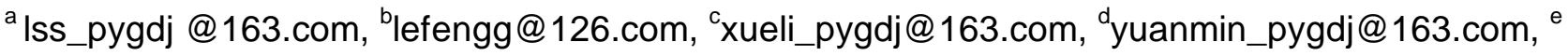 \\ zjli@huatianpower.com
}

Keywords: distribution network automation; distributed feeder automation; smart distribution transformer terminal; IDTT-B; GPRS/CDMA communication

\begin{abstract}
In distribution automation systems, transformer terminal unit (TTU) which, as a basic unit was distribution measurement and control equipment and was installed in branched distribution box of power basement and communicated with power main station through the communication network. Its performance determined and had a direct impact on whether the whole distribution automation system could work effectively. A new type of wall mounted intelligent distribution terminal equipment was designed for distribution network's operation and management, and which integrated distribution transformer monitoring, power quality monitoring, temperature and humidity measurement, and low voltage switching communication. It also had high degree of integration, and advanced technique, and was powerful, and could support GPRS/GSM wireless communication mode and remote control, remote communication and remote software upgrade, so as to realize the upgrading of distribution area, with low investment and high cost performance.
\end{abstract}

\section{Introduction}

The transformer terminal unit (TTU) is terminal device which is installed next to the distribution transformer to monitor the operation status of transformer ${ }^{[1]}$. TTU is used to perform information acquisition and control on transformer and monitor the transformer's operation situation, so as to realize the functions of voltage meter, power meter, power factor meter, load indicator ad voltage monitoring instrument. It can communicate with other background equipment, and provide the data needed in controlling and managing in distribution system. Generally, it's required to monitor the operation of lines, distribution transformer or box type transformer, and timely find and deal with the accident and emergency situation, and carry out local or remote reactive compensation, as well as to realize automatic voltage regulation of on-load tap changer (OLTC) ${ }^{[2]}$.

The development of distribution monitoring system has been a considerable scale in foreign countries. Its development has experienced the process from a variety of single automatic equipment in distribution system to an open, integrative, and network integrated of comprehensive automatic distribution system. Currently, the foreign research is focused on single distribution automatic expert system and distribution network training system, and is studying the optimization of load distribution to reduce the network loss, and performs management on transformer load, and so as to take use of transformer capacity to the fullest and reduce the reactive power loss of system as well as manage the electricity users' load according to time price ${ }^{[3,4]}$.

The distribution monitoring system has been carried out pilot work in many domestic power companies, but the main problems exist in current distribution monitoring system are, on one hand, the distribution points of distribution transformer are many and its distribution range is wind, which causes it is difficult to select the appropriate communication mode, and so that the information cannot be delivered effectively; on the other hand, because of the lack of effective analysis software of distribution transformer, then the distribution transformer information cannot be collected and applied 
effectively. It causes that although a part of power distribution transformer monitoring terminal is put into operation in the field, but the effect is not ideal, and the transformers are failed to get running state analysis and economic operation analysis ${ }^{[5,6]}$.

In view of the above limitations, and based on the current situation and demand of domestic distribution network, a new type of wall hanging intelligent terminal device for distribution operation and management is introduced in the thesis, which integrates the functions of distribution transformer monitoring, power quality monitoring, measurement of temperature and humidity, and low voltage switch communication, and it has high integration degree, advanced technique, and high performance, and supports GPRS / GSM wireless communication and remote software upgrading, so as to realize the transformation and upgrading of distribution area, with low investment and high ratio of performance to price.

\section{System design principle}

The IDTT-B type of distribution terminal is composed of nine parts: the main control module, communication module, the input $\mathrm{AC}$ acquisition module, the output $\mathrm{AC}$ acquisition module, DC analog signal acquisition switch, reactive power compensation control module, input and output module, carrier wave communication module and power supply module. The principle diagram is shown in Fig.1.

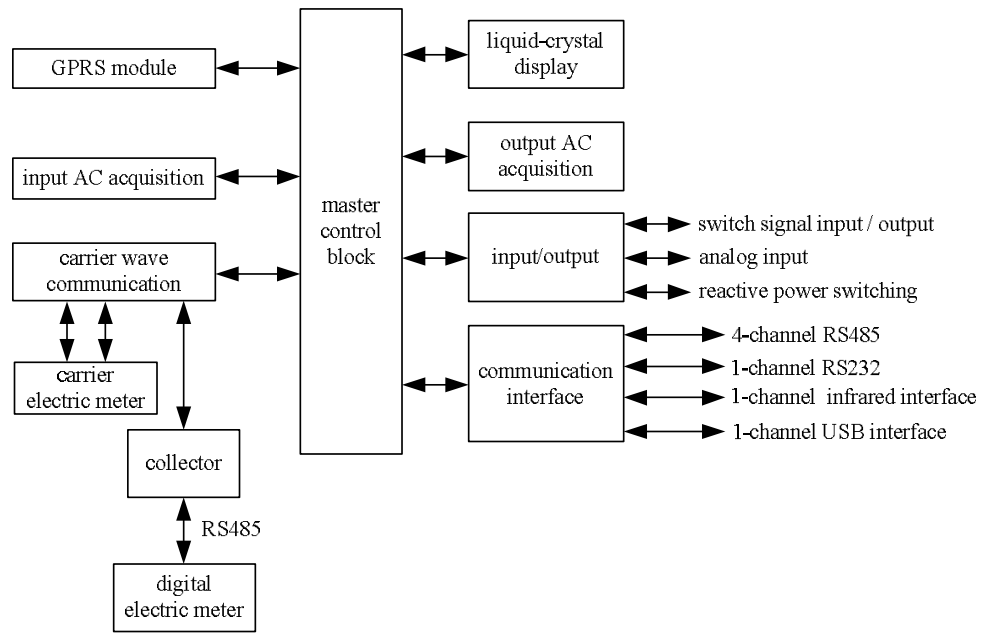

Fig.1 Block diagram of system

Signal acquisition module. (1) State variables acquisition circuit. The state variables monitored by TTU are actually the on-off state of the passive switches, and the state variables acquisition circuit is shown in Fig.2, in which, one end of passive switch is connected to the ground for isolation, and the other end is connected to the cathode of the optical coupler, then it can be switched on, and its TEST end outputs low level; If the switch is disconnected, the optical coupler is open, and its TEST end outputs high level. The passive switch state can be determined by monitoring the level of TEST end by CPU.

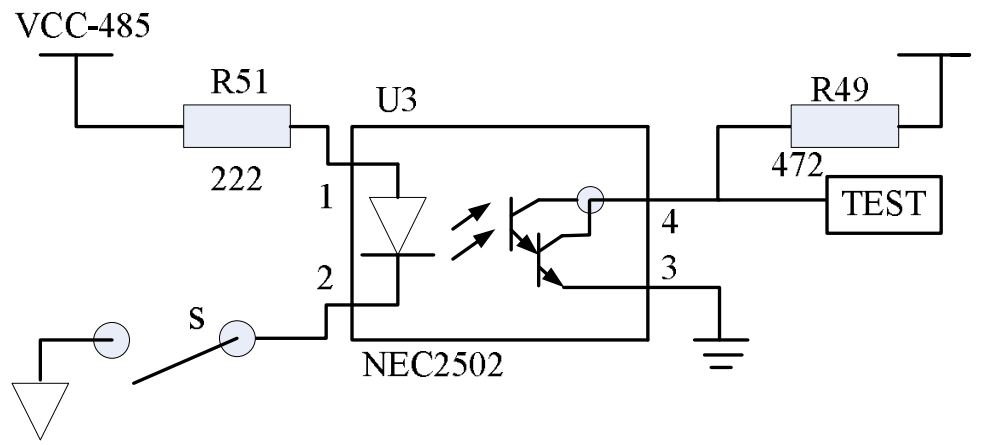

Fig. 2 Switch variables acquisition circuit 
In order to avoid the false judgment of impacts of interference signal on the switching state, the software design needs to take the software filter into consideration, and the most commonly used method is to read the average value.

(2) Analog variables acquisition circuit. The analog variables acquisition function of the monitoring terminal is actually to obtain the DC analog voltage/ current values output by the device. The acquisition ranges of two channels of voltage and current are required in the TTU, and the voltage range is $0-5 \mathrm{~V}$, the current range is $4-20 \mathrm{~mA}$.

The analog variables acquisition circuit is shown in Fig.3, in which, the voltage and current signal firstly pass through the signal conditioning circuit, and them converts into the voltage signal which can be received ty ADC0834 chip, and then sends to the ADC0834 for AD conversion, and finally, the conversion results are transmitted to the STM32 chip ty the four lines SPI. In order to improve the reliability of terminal, it requires electrical isolation between the analog input and the main control circuit, in view of low speed of optical coupler, and needs external devices, and larger area of circuit board, therefore, the RF coupling high speed isolation chip Si8441 of Silicon Libs is selected for design in this thesis.

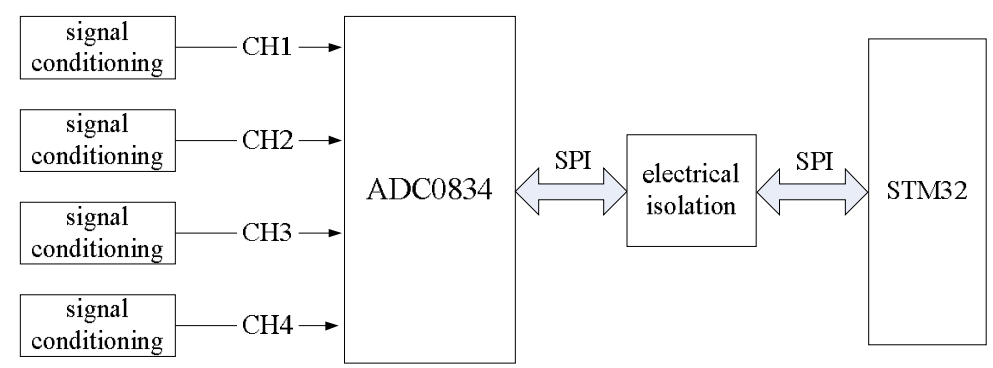

Fig.3 Analog variables acquisition circuit schematic diagram

The RF coupling mode is adopted in Si8441 to realize digital signal isolation, and the current isolation and durability reaches to $2500 \mathrm{~V}_{\text {RMS }}$. The Si84441 can transfer high frequency signal which is up to $100 \mathrm{Mhz}$, and the power consumption is lower than the optical coupler, but also transmit a high resistance state of the device. It also doesn't need external devices, and a single chip can achieve the isolation of 4 channels digital signal. For the cost, it is equivalent with the price of high speed optical coupler ${ }^{[7]}$.

AC sampling module. The AC sampling module is divided into the input $\mathrm{AC}$ acquisition module and output $\mathrm{AC}$ acquisition module, and respectively collects the voltage and current of inlet and outlet, and then are sampled by the sampling circuit and sent to the special metering chip, and then the obtained basic parameters are transmitted to the processing module. Each kind data is performed statistical analysis by the processing module and then solved in the flash chip of processing module and finally waits for measuring by the main station. Meanwhile, the processing module also performs the commands from main station. The processing module is also equipped with a standard RS232 interface and infrared interface for on-site commissioning and maintenance ${ }^{[8,9]}$.

\section{System performance}

The IDTT-B smart transformer terminal introduced in this thesis is a new generation of distribution transformer monitoring optimization terminal which is based on the development of embedded hardware and software platform, and which is also a new developed generation of equipped with electric product according to the application requirements of domestic distribution and electricity using departments.

The terminal has high integration level, advanced technique, and strong function. It is used in the condition monitoring and power management of distribution transformer, and adopts GPRS/ CDMA wireless communication mode, and which is widely used in rural power network and urban low voltage power distribution network. The terminal performs all kinds of data collection, analysis, and storage of the public distribution station area, and can perform reactive power compensation and power quality 
monitoring automatically based on real-time data from the power grid. It also performs data monitoring and controlling on the low voltage residual current circuit breaker by means of communication. It performs the electric meter transcribing of residents through carrier communication; it supports emote communication, remote software update and summary transcription, and so on, which is a low-voltage distribution product with a collection of many kinds of functions. Its main functions are shown as follows.

1) Real time power distribution monitoring. Can monitor three-phase voltage, current, active power, reactive power and power factor, etc. in real time; display a variety of electric energy data, such as power, the rate of electric energy, the total power, etc.; make the statistics of qualified rate of voltage, three-phase imbalance, harmonics, voltage fluctuations and flicker, etc.; monitor the state transition of capacitors and operating state of distribution terminals.

2) Distribution transformer protection. The device has functions of over voltage protection, over current protection, over load protection, and over heat protection and so on.

3) Electricity using information monitoring. Can collect the user's power consumption, and the capacity data of power distribution transformer, and carry out abnormal operating condition analysis and alarm, etc.

4) Load management. Closed loop control for power, power control, and remote control.

5) Power quality management. Reactive power compensation, three phase unbalance, and harmonic control.

6) Security protection. Has a function of anti-theft, electricity-stealing prevention, hardware security module, and abnormal information alarms, etc.

7) Control ability. Low voltage outlet switch control, reactive power compensation control, load control, sound and light emitting device control.

8) Communication function. GPRS/CDMA uplink communication, carrier wave and RS485 downlink communications; the terminal has 4 channels of RS485 communication interface, forms electrical isolation between the interface and internal circuit, palm computer field management, local infrared, and RS232 communications management ${ }^{[10,11]}$.

\section{Technique characteristics of the system}

The characteristics can be summarized as follows.

1) The master CPU uses the embedded 32 bit system, with the real-time processing ability; and equipped with power RAM and high performance CPU monitor chip. The device is stable and reliable, and it will not lose information without power. Using the page management technology, with a large capacity flash memory and can carry out a large number of data storage, and before receiving the refresh command, the terminal can both save the data regardless of power outage.

2) It is fully coincidence in the requirements of the national newly promulgated DL/T7376.1 "system communication protocol of power users' electricity information acquisition" on the terminal. It supports DL/T645-1997/2007 multi-function electric energy meter communication protocol, and the industry's variety of domestic and exterior design agreement.

3) It takes use of the TCP/IP and FTP protocol library, and the network speed of terminal is faster, and the network process is controllable, and supporting local and remote software and meter specification library online upgrade, and the local upgrade through the USB interface, and the remote upgrade is optional of FTP or TCP/IP approach, with a high speed of upgrading.

4) Modular design, perfect interface, flexible configuration of communication module (optional), supports GPRS/CDMA Ethernet, supports for the integration of communications, built-in and external mode variable.

5) Perfect electromagnetic compatibility and reliability design, double watchdog circuit, to ensure the safe and reliable operation of the terminal and easy to maintain.

6) Special configuration of DC sampling performs the acquisition of PT100 sensor or 4-20mA signal, easy to collect the parameters of transformer. 
7) Adopt high precision metering chip, with high precision, fast speed, and complete parameters, and supports 19 times of harmonic acquisition.

8) Specially designed power supply module, with wide voltage input, and use three-phase power supply, and can word normally in lack of two phases.

9) Take use of high accuracy and real-time clock module in no power off.

10) The terminal has the function of data concentrator meter.

11) The terminal has a very strong self-test function.

12) Reactive power switching function, multi group of capacitors are worked in grouping and circulating switching, and according to the national standard, the reactive power as the basis for switching.

13) The terminal is configured with a multi-channel communication interface, and the internal configuration of communication can be used, and also can configure the external to do a transparent forwarding, to facilitate communication between users.

\section{Application}

IDTT-B intelligent distribution transformer terminal adopts advanced control and network communication technique and can conveniently realize the collection, processing and control of information in city and rural distribution area. The terminal has a high integration and a small size, and is easy to install, and can be integrated into the JP cabinet and box type of power distribution cabinet.

The intelligent distribution terminal supports GPRS/CDMA communication and supports RS-485, carrier wave and wireless communication interface. It also supports the "electric power users information acquisition system communication protocol (DL/T376.1)", and the "multi-function electric energy meter communication protocol (DL/T645-1997/2007) ". The Fig.4 is a structure schematic diagram of the intelligent terminal device is access to the smart station area monitoring system. Select a distribution area in a certain place, and take use of smart distribution area monitoring equipment to achieve the upgrading of the original distribution area (as is shown in Fig. 5).
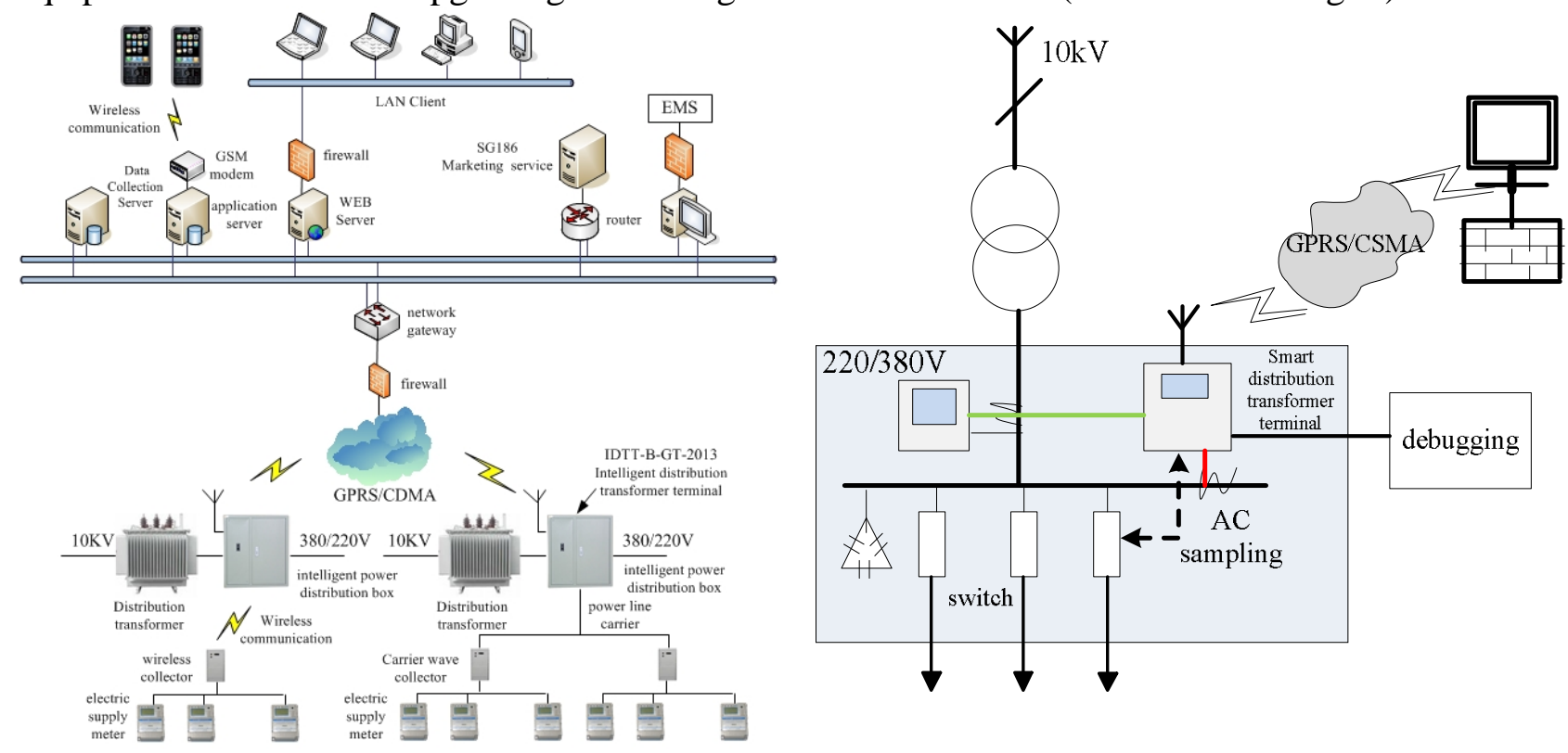

Fig.4 The schematic diagram of the TTU connecting to the intelligent station

Fig.5 Upgrading diagram of original distribution area

The production, management, analysis and maintenance in distribution area are achieved by the operational and management system. And the functions of the distribution transformer monitoring, smart switch control and parameter management in distribution area are realized by the intelligent distribution terminal, which is shown in Fig.6. 


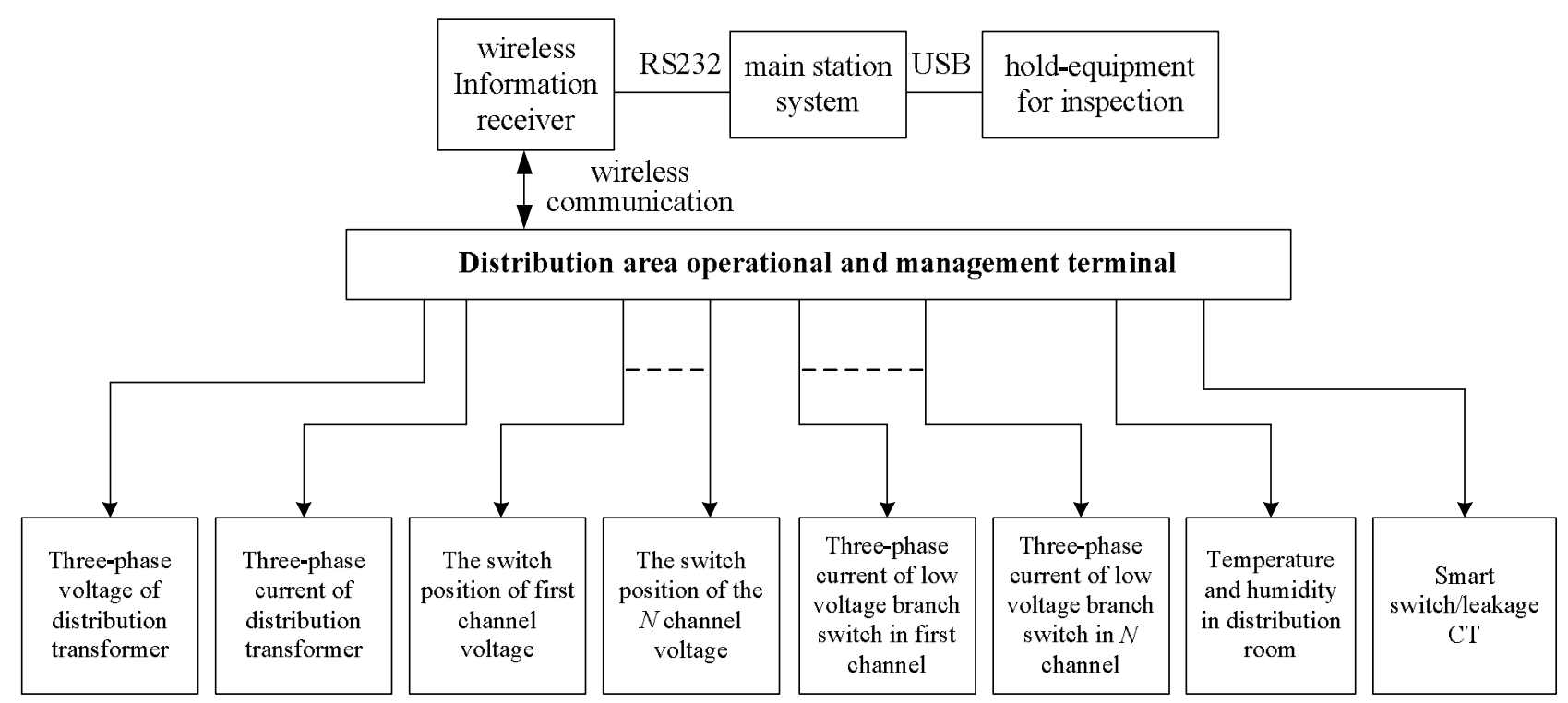

Fig.6 The working principle diagram of distribution transformer terminal

The distribution area operational and management terminal can directly collect $3 I$ and $3 U$, and the distribution transformer operational data which including the active power, reactive power, power factor and zero sequence current are calculated according to the AC sampling results.

If the switch takes use of intelligent residual current breaker, then the state of the switch and the leakage value can be transferred to the master station system through the intelligent distribution terminal. If there is no intelligent residual current breaker, the state of the switch is acquired through the DI interface, and the leakage value is measured by the leakage CT which is installed into the intelligent distribution terminal, and then transmitted to the main station system.

The information such as the temperature and humidity of the distribution station is connected with the AI and DI interface, and is sent to the master station system through the intelligent distribution terminal.

The inspection is performed by taking use of the hand-held equipment (PDA or smart phone) + locating points (two-dimensional code), after the inspection is completed by the personnel inspection, then the smart handheld devices are access into the computer, and import data into the main station system through the USB approach to make statistics and analysis of the inspection situation.

\section{Summary}

(1) Based on the current situation and demand of distribution area, a new wall type of smart distribution area operational and management terminal device was introduced, which took use of smart components and modular application, and integrated multi functions of distribution transformer monitoring, harmonic analysis, centralized meter reading, reactive power compensation, remote communication and temperature and humidity measurement. Each application module was independent in design and function according to the component-based design of various functions of the components and the design of intelligent bus and uniform interface, so that achieves plug and play applications.

(2) In practical application, the smart terminal can be configured different application module s according to the field demand to complete the required functions. o that the terminal not only has good cost performance, but also has good expansibility, which is of great significance to realize the intelligence of distribution area and provide effective monitoring data for the operating maintenance, and provide effective technology guarantee for the operation and management of distribution network, as well as improve the safety, stability and reliability of the distribution network. 


\section{References}

[1] Bingyin Xu, Tianyong Li, Yongduan Xue, Smart distribution grid and distribution automation, J. Automation of Electric Power Systems, 2009, 33 (17): 38-41.

[2] Yefei Zhou, Shiming Xu, A preliminary investigation on data center in smart substation, J. Automation of Electric Power System, 2011, 35 (18): 57-61.

[3] Bingbing Shen, Lin Wu, Peng Wang, Technological characteristics and application effects analysis of distribution automation pilot projects, J. Automation of Electric Power Systems, 2012, 36 (18): 27-32.

[4] Guopei Wu, Yuquan Liu, Research and application of technology support system for smart distribute grid, J. Power System Protection and Control, 2010, 38(21): 162-166+172.

[5] Jianping Cao, Ying Ni, Situation and development of distribution transformer terminate unit, J. Electrical Applications, 2005 (1): 18-21.

[6] Jian Yang, Key technology research of reactive compensation system in distribution network, D. Changsha: CENTRAL SOUTH UNIVERSITY, 2002.

[7] Weibing Liu, Research of rural smart distribution district typical construction scheme, D. Baoding: North China Electric Power University, 2012.

[8] Wenzhao $\mathrm{Wu}$, Research on rural smart distribution area construction, J. Electrical Engineering, 2013, S1:34-36+41.

[9] Xinhe Chen, Wei Zhao, Xing Zhang et al, Application function and configuration of smart distribution station, J. Rural Electrification, 2014, 02:37-38.

[10] Wenliang Zhang, Zhuangzhi Liu, Mingjun Shi, et al, Development trend of smart grid, J. Power System Technology, 2009, 33 (13):1-11.

[11] Shuyi Yu, Kai Yin, Application of intelligent remote monitoring system in low voltage distribution network, J. Hu Bei Power, 2011 (6):43-45. 